\title{
AVALIAÇÃo DA IMPLANTAÇÃo DA POLÍTICA DE SAÚDE DA POPULAÇÃo NEGRA NA BAHIA
}

\author{
Adivânia Tolentino Nogueira ${ }^{1}$; Edna Maria de Araujo²; Silvone Santa Bárbara da \\ Silva $^{3}$; Tarcísio Oliveira Silva ${ }^{4}$, Kevin Góes Lôbo ${ }^{5}$ \\ 1. Bolsista PIBIC/CNPq, Graduando em Medicina, Universidade Estadual de Feira de Santana, e-mail: \\ adivania.nogueira@gmail.com \\ 2. Orientador, Departamento de Saúde, Universidade Estadual de Feira de Santana, e-mail: ednakam@gmail.com \\ 3.Participante, Professora Adjunta do Departamento de Saúde- UEFS, email: silvone.santabarbara@gmail.com \\ 4. Participante, Discente do Mestrado Profissional em Enfermagem - UEFS email: tosbahia@gmail.com \\ 5. Participante, Graduando em Medicina na UEFS, email: keevinlobo@hotmail.com
}

PALAVRAS-CHAVE: Políticas de Saúde, População Negra, Indicadores de Saúde

\section{INTRODUÇÃO}

A Política Nacional de Saúde Integral da População Negra foi aprovada no ano de 2006 pelo Conselho Nacional de Saúde, como um instrumento que tem por objetivo combater a discriminação étnico-racial nos serviços e atendimentos oferecidos no Sistema Único de Saúde, bem como promover a eqüidade em saúde da população negra (Ministério da Saúde, 2007).

O exercício da prática desta política é uma importante contribuição para a consolidação do Sistema Único de Saúde, garantido o direito universal à saúde como uma condição para a democracia participativa. É igualmente coerente com o objetivo fundamental da República Federativa do Brasil de "promover o bem de todos, sem preconceitos de origem, raça, sexo, cor, idade e quaisquer outras formas de discriminação" (BRASIL, 1988).

Segundo Araújo et al. (2010), a formação do campo da saúde da população negra ocorre no momento em que o debate sobre o racismo e a formulação de programas de ação afirmativa assume crescente visibilidade no cenário brasileiro. As principais queixas a respeito do acesso e da qualidade da atenção à saúde referidas pela população negra são: dificuldade de acesso, assistência não-resolutiva, preconceito racial, preconceitos com a pobreza, maus-tratos, preconceitos relacionados à prática de religião afro-brasileira e "invisibilidade" (Gomes, 2010), ou seja, a pessoa frequenta os serviços, mas a cada consulta é visualizada como se fosse o primeiro atendimento, desconsiderando a necessidade da continuidade do cuidado.

Acreditando na superação destas dificuldades através da aplicação da PNSIPN, este trabalho analisou indicadores de acesso as ações e serviços de saúde, com recorte étnicoracial, no Estado da Bahia, utilizando os resultados desta análise como referência de transformações que podem ser alcançadas na reorganização da atenção à saúde, através da adoção de ações institucionais globalizantes e includentes.

\section{MATERIAL E MÉTODOS}

A pesquisa se desenvolveu no campo da análise de implantação da Política Nacional de Saúde Integral da População Negra nos municípios da Bahia no período de 2010-2014. O lócus do estudo foram os municípios do estado da Bahia, estratificados por regiões de saúde e que possuíam comando único.

Utilizou-se a abordagem quantitativa com ampla revisão documental, com base no plano de indicadores da dimensão a acesso aos serviços de saúde. Foram identificados e lidos aproximadamente 90 documentos de relatórios de gestão, entre os anos de 2010 e 2014, sendo 
esses relatórios produzidos no âmbito municipal e estadual (Secretaria de Saúde da Bahia). Além de 8 relatórios epidemiológicos de morbimortalidade e assistência à saúde divulgados pelo Sistema do DATASUS. Fizeram parte da pesquisa 80 municípios.

$\mathrm{Na}$ análise dos dados identificou-se os municípios que apresentaram o grau mais avançado na implantação da PNSIPN. A análise foi realizada por municípios e os indicadores foram analisados separadamente, com apresentação de tabelas e gráficos feitos no EXCEL. Observou-se a tendência do comportamento dos indicadores, isto é, se existiu uma diferença anual no período de 2010-2015. Depois fez-se uma descrição do percentual por indicador dos municípios que alcançaram a meta pactuada para cada indicador.

Essa pesquisa obedeceu aos princípios éticos da pesquisa, e foram adotadas as recomendações do Conselho Nacional de Pesquisa (CONEP).

\section{RESULTADOS E/OU DISCUSSÃO}

Os resultados foram apresentados por indicador, para observar em qual área a PNSPN teve maior avanço e quais foram os municípios em que esses avanços ocorreram. Depois os municípios que alcançaram os parâmetros dos indicadores foram reunidos para identificar aqueles com maior número de indicadores alcançados, permitindo nomear os municípios onde a implantação da PNSIPN está mais avançada.

Observou-se diferentes situações em cada indicador e em cada ano analisado. De um modo geral, na análise comparativa do percentual de municípios que alcançaram os parâmetros dos indicadores, não se observou um município que alcançasse todas as metas. Os dados do estudo evidenciaram que não houve avanços consideráveis na melhoria do acesso as ações e serviços de saúde oferecidos à população negra em nenhum município. E consequentemente, que há dificuldades na implantação da PNSIPN. Isso é visto mais claramente quando durante a pesquisa nos Relatórios Municipais de Saúde (que descrevem as ações e serviços de saúde realizados nos municípios) em nenhum momento é proposta ações que apoiem a implantação da política. Já nos Relatórios de Gestão Estadual da Bahia, a Política é citada, e ações para capacitação de profissionais de saúde são divulgadas, assim como encontros e projetos com Comunidades Quilombolas e de Religião de Matriz Africana. As ações foram descritas como visitas técnicas e principalmente capacitação de profissionais. Ou seja, pode-se inferir que no Estado da Bahia a responsabilidade pela implantação da PNSIPN está sendo muito mais debatida em âmbito de gestão estadual do que de gestão municipal.

No que diz respeito ao indicador de acompanhamento da gestação de mulheres negras e pardas, com consultas pré-natal em número de 6 consultas, 7 ou mais consultas, conforme preconiza o Ministério da Saúde, dos 80 municípios, apenas 23 elevaram em 20\% o percentual de consultas pré-natais:

Tabela 1 - Municípios selecionados da Bahia com elevação na Proporção (\%) de Nascidos Vivos segundo o número de consultas de pré-natal (4 a 6 consultas, 7 ou mais consultas) e raça/cor parda/preta. Municípios da Bahia, 2010-2014. Fonte: SINASC

\begin{tabular}{|c|c|c|c|c|c|}
\hline Região de Saúde/Município & 2010 & 2011 & 2012 & 2013 & 2014 \\
\hline ..... Crisópolis & $28 \%$ & $37 \%$ & $56 \%$ & $54 \%$ & $57 \%$ \\
\hline ..... Livramento de Nossa & $65 \%$ & $77 \%$ & $79 \%$ & $77 \%$ & $86 \%$ \\
\hline Senhora & & & & & \\
\hline ..... Sapeaçu & $30 \%$ & $54 \%$ & $57 \%$ & $66 \%$ & $69 \%$ \\
\hline ..... Santo Estêvão & $33 \%$ & $40 \%$ & $62 \%$ & $47 \%$ & $53 \%$ \\
\hline ..... Caetité & $51 \%$ & $73 \%$ & $73 \%$ & $76 \%$ & $81 \%$ \\
\hline
\end{tabular}




\begin{tabular}{l|lllll}
\hline .... Ibotirama & $57 \%$ & $68 \%$ & $69 \%$ & $72 \%$ & $76 \%$ \\
.... Ibicaraí & $35 \%$ & $54 \%$ & $46 \%$ & $51 \%$ & $57 \%$ \\
.... Itabuna & $34 \%$ & $47 \%$ & $49 \%$ & $51 \%$ & $55 \%$ \\
.... Capim Grosso & $38 \%$ & $53 \%$ & $61 \%$ & $60 \%$ & $67 \%$ \\
.... Morro do Chapéu & $41 \%$ & $51 \%$ & $60 \%$ & $65 \%$ & $66 \%$ \\
.... Jeremoabo & $38 \%$ & $49 \%$ & $65 \%$ & $60 \%$ & $69 \%$ \\
.... Itabela & $44 \%$ & $45 \%$ & $62 \%$ & $59 \%$ & $74 \%$ \\
.... Bom Jesus da Lapa & $26 \%$ & $37 \%$ & $56 \%$ & $60 \%$ & $63 \%$ \\
.... Laje & $26 \%$ & $39 \%$ & $35 \%$ & $39 \%$ & $50 \%$ \\
.... Santo Antônio de Jesus & $39 \%$ & $62 \%$ & $64 \%$ & $60 \%$ & $62 \%$ \\
.... São Felipe & $28 \%$ & $44 \%$ & $56 \%$ & $53 \%$ & $61 \%$ \\
.... Cansanção & $21 \%$ & $41 \%$ & $56 \%$ & $59 \%$ & $63 \%$ \\
.... Euclides da Cunha & $59 \%$ & $46 \%$ & $63 \%$ & $60 \%$ & $66 \%$ \\
.... Monte Santo & $25 \%$ & $39 \%$ & $45 \%$ & $50 \%$ & $56 \%$ \\
.... Santaluz & $34 \%$ & $51 \%$ & $59 \%$ & $57 \%$ & $64 \%$ \\
.... Belo Campo & $48 \%$ & $66 \%$ & $60 \%$ & $58 \%$ & $76 \%$ \\
.... Maetinga & $38 \%$ & $51 \%$ & $55 \%$ & $63 \%$ & $65 \%$ \\
.... Presidente Jânio Quadros & $37 \%$ & $50 \%$ & $59 \%$ & $57 \%$ & $71 \%$ \\
\hline
\end{tabular}

Fonte: SINASC/ DATASUS

A ineficácia dos programas de ampliação do Pré-natal para a população negra foi também confirmada quando se analisou o indicador de relação de pré-natal insuficiente entre negras e não negras nos Municípios da Bahia, e observou-se que as situações mais desfavoráveis e de maior vulnerabilidade são mais frequentes em pardas e pretas, sendo estas mulheres as que mais realizam pré-natal insuficiente quando comparado com as mulheres não negras. Apenas nos municípios de Brumado e Lauro de Freitas esta relação diminuiu entre 2010 e 2014.

Outro indicador analisado foi a oferta de leitos neonatais intensivos com objetivo principal de reduzir a mortalidade neonatal. Considerando que a Bahia tem uma população de maioria negra, deu-se importância para avaliar as condições de serviços oferecidos a população em geral como parâmetro para inferir a qualidade do serviço de UTI neonatal nos serviços públicos, considerando a população negra como principal usuária do serviço público de saúde. Dos 80 munícipios, 11 possuíam leitos de UTI neonatal, e destes apenas Camaçari e Juazeiro apresentaram proporção inferior a esperada para a quantidade de leitos: menos de 2 leitos de UTI neonatal para cada 1.000 nascidos vivos na região (CNES,2015). Os demais municípios superaram a quantidade esperada, o que sugere melhora na qualidade do atendimento com cuidados intensivos à população neonatal, e consequentemente para a população negra dessa faixa etária.

Com relação ao indicador vigilância do óbito infantil na população negra nos municípios de Itabela e Amargosa, o número de óbitos diminuíram de forma linear no intervalo de tempo considerado. Porém, na maioria dos municípios da pesquisa, com destaque para Salvador, que registrou o maior número de óbitos em todos os anos da série histórica, os valores não sofreram alterações ou mantiveram oscilações de um ano para outro. Deve-se atentar, contudo, que o aumento no número de óbitos infantis nos municípios pode ser devido a melhoria na alimentação dos Sistema de informação em relação a um ano para o outro.

Para os indicadores Razão da mortalidade por doenças diarreicas ou pneumonia por raça/cor), nos municípios que tinham completude dos dados na série histórica (dados do SIM/DATASUS, observou-se maior proporção de óbitos infantis de pardos e negros, em 
muitos com $100 \%$ dos óbitos, sinalizando tanto diferenciais nos níveis de mortalidade como nas condições socioeconômicas, entre brancos e negros. Sinaliza-se que, em boa parte dos municípios que foram lócus desta pesquisa não há registros na base de dados do SIMDATASUS, isso pode ter ocorrido por falha no preenchimento das declarações de óbito ou por inadequada alimentação do sistema de base de dados.

Quanto a Cobertura vacinal com a Pentavalente em crianças dos Municípios com comunidade quilombola registrada no Portal Palmares, nos dados dos relatórios municipais de gestão de saúde nenhum desses municípios alcançou a meta de $100 \%$ de vacinação na população da amostra. Esse registro leva a inferir que em muitas comunidades quilombolas o serviço de imunização infantil de menores de 1 ano ainda não é satisfatório. É necessário intensificar as campanhas e aproximar os serviços de saúde da população quilombola, ampliando os serviços de saúde ofertados a este grupo populacional.

O que se percebeu a partir desse estudo corroborou com a perspectiva de SILVA et al. (2016), de que existem muitos entraves que dificultam a implantação e manutenção de programas de saúde pública no Brasil, mas que ao se produzir informações sobre os processos de implementação e seus resultados, podem-se elaborar e propor soluções criativas e focadas na construção de serviços mais eficientes.

\section{CONSIDERAÇÕES FINAIS}

Em síntese, a implantação da Política de Saúde da População Negra na Bahia enfrenta muitas problemáticas. Pelos indicadores de acesso ao serviço de saúde e pelos registros dos Relatórios Estaduais e Municipais de Saúde, percebe-se que após cinco anos da criação da PNSIPN, nos municípios Baianos com comando único a política não está implantada sendo que em nenhum dos relatórios municipais há menção a ações que viabilizem esta implantação, e em relação aos indicadores não houve avanços significativos na melhoria do acesso aos serviços de saúde na maioria dos municípios. Espera-se que os indicadores aqui apresentados possibilitem melhor orientação da prestação de serviços, objetivando o alcance de maior equidade e afirmação da necessidade da implantação da PNSIPN.

\section{REFERÊNCIAS}

Araújo, Carla Luzia França; Cruz, Luciane Maria França da; Lopes, Márcia Cristina; Fernandes, Elizabeth Cristina da Silva. O quesito cor/raça em formulários de saúde: a visão dos profissionais de saúde. Rev. enferm. UERJ, Rio de Janeiro, V. 18, n. 2, p. 241-6, abr/jun. 2010.

Brasil. Ministério da Saúde (MS). Política Nacional de Saúde Integral da População Negra.1. Brasília: MS; 2007

BRASIL. Constituição (1988). Constituição da República Federativa do Brasil. Brasília, DF: Senado, 1988.

GOMES, Márcia Constância Pinto Aderne. Projeto: Ylê ayié yaya ilera (Saúde plena na casa desta existência): equidade e integralidade em saúde para a comunidade religiosa afro-brasileira. Interface (Botucatu), Botucatu , v. 14, n. 34, p. 663-672, Sept. 2010. Disponível em $<\mathrm{http}: / / \mathrm{www}$.scielo.br/scielo.php?script=sci_arttext\&pid=S1414-

$32832010000300015 \& 1 n g=$ en\&nrm=iso $>$. Acesso em 12 Set. 2016.

SILVA, Regina Lúcia Dalla Torre et al . Avaliação da implantação do programa de assistência às pessoas com hipertensão arterial. Rev. Bras. Enferm., Brasília , v. 69, n. 1, p. 79-87, Feb. 2016. Available from $\quad<$ http://www.scielo.br/scielo.php?script=sci_arttext\&pid=S003471672016000100079\&lng=en\&nrm=iso>. Acesso em 24 Mar. 2016. 